\title{
COMPONENTES DEL CONTROL INTERNO EN PEQUEÑAS Y MEDIANAS EMPRESAS
}

\section{COMPONENTS OF INTERNAL CONTROL IN SMALL AND MEDIUM-SIZED ENTERPRISES}

\author{
Rojas-Salvatierra Wellington ${ }^{1 *}$; Chiriboga-Mendoza Marola²; Pacheco-Vergara Javier ${ }^{3}$ \\ ${ }^{1}$ Universidad Laica Eloy Alfaro de Manabí, ULEAM. Manta, Ecuador. \\ ${ }^{2}$ Directora Financiera de la Fundación Crecer. Manta, Ecuador. \\ ${ }^{3}$ Universidad Laica Eloy Alfaro de Manabí, ULEAM. Manta, Ecuador.
}

*Correo: wars11_5_94@gmail.com

\begin{abstract}
Resumen
El control interno ha venido potenciando su importancia en las organizaciones que tienen la necesidad de controlar sus pertenencias y las del grupo que integran las entidades. Los cambios estructurales y tipológicos de las empresas han ocasionado que se generen nuevas medidas y estrategias para adaptarse a las necesidades del medio. Un sistema de control interno abarca aquellos aspectos que se relacionan directamente con la función de los departamentos de contabilidad y finanzas para incluir el control de presupuestos, costos estándar, reportes periódicos relacionados con las operaciones y análisis estadísticos. Por lo tanto, la implantación del control interno se realiza mediante un sistema integrado que involucra diversos aspectos, tales como: principios, normativas, procedimientos y metodologías. Este manuscrito tiene como objeto revisar la literatura referente al control interno aplicado en pequeñas y medianas empresas. Aspectos importantes en la definición del control interno refieren que, a efecto de las pymes, el control interno se basa en un procedimiento ejecutado por personas a partir de la implantación de procedimientos estandarizados que contribuyen con la consecución de los objetivos empresariales.
\end{abstract}

Palabras clave: control financiero, pyme, principios, estructura empresarial.

\begin{abstract}
Internal control has been enhancing its importance in organizations that need to control their belongings and those of the group that make up the entities. The structural and typological changes of the companies have caused new measures and strategies to adapt to the needs of the environment. An internal control system encompasses those aspects that are directly related to the function of the accounting and finance departments to include control of budgets, standard costs, periodic reports related to operations and statistical analysis. Therefore, the implementation of internal control is done through an integrated system that involves various aspects, such as: principles, regulations, procedures and methodologies. The purpose of this manuscript is to review the literature regarding internal control applied in small and medium-sized companies. Important aspects in the definition of internal control refer that, for the purposes of SMEs, internal control is based on a procedure executed by people based on the implementation of standardized procedures that contribute to the achievement of business objectives.
\end{abstract}

Keywords: financial control, SMEs, principles, business structure.

Información del manuscrito:

Fecha de recepción: 02 de mayo de 2018

Fecha de aceptación: 28 de junio de 2018

Fecha de publicación: 10 de julio de 2018 


\section{Introducción}

Los cambios estructurales y tipológicos de las empresas han ocasionado nuevas medidas $y$ estrategias para adaptarse a las necesidades. Las empresas estadounidenses y de países europeos han asumido procedimientos y criterios para adaptarse a la nueva dinámica que exige el control de las empresas en función del riesgo empresarial (Andrés et al., 2012).

El control interno ha venido potenciando su importancia en las organizaciones que tienen la necesidad de controlar sus pertenencias y las del grupo que integran las entidades, Ricard (2017) concluye que desde la economía pre capitalista, con los modos de producción esclavista y feudal, el control y la revisión se establecen como instrumentos de la clase dominante en función de sus intereses.

Nagua \& Burgos (2016) explican acerca del control interno en pequeñas y medianas empresas de carácter familiar, como una oportunidad para mejorar la competitividad empresarial, además, mencionan en sus conclusiones que el control interno es un proceso efectuado por la máxima autoridad y los demás miembros de la organización, cuyo objetivo es obtener un respaldo seguro de todas las actividades, optimizando la gestión y manejo administrativo. Asimismo, las pequeñas y medianas empresas que realizan un control interno desempeñan un rol esencial en la sociedad ya sea produciendo $u$ ofertando bienes o servicios a partir de un sistema debidamente establecido y estructurado que genera significativas ventajas $y$ permite salvaguardar los activos de la empresa.

Hoy en día las pymes se han convertido en un elemento clave del crecimiento de la economía. En Latinoamérica, las pymes presentan una estructura caracterizada por escalas a baja producción, aplicación de tecnologías adaptadas, con escaso acceso a fuentes de financiamiento (Andrés et al., 2012). Guachi (2017) reporta acerca de un análisis del sistema de control interno y su impacto en el proceso de ejecución presupuestaria del Jipijapa, Manabí, Ecuador; y entre sus recomendaciones indica que el control interno es la 
herramienta de toda actividad administrativa financiera de las instituciones que administran y manejan recursos financieros, por consiguiente, un mayor control financiero requiere que se estandaricen las auditorías, y consecuentemente se mejora la calidad del gasto público. Aspectos importantes en la definición del control interno refieren que, a efecto de las pymes, el control interno se basa en un procedimiento ejecutado por personas a partir de la implantación de procedimientos estandarizados que contribuyan a la consecución de los objetivos, pero garantizando la prevención de los riegos internos y externos a los que se enfrenta la empresa.

En la actualidad, muchas empresas mantienen un control interno establecido no solo por aspectos de auditoría, sino que han considerado relevante su aplicación con el fin de establecer lineamientos por medio de normas y procedimientos, las mismas que sirven de guía para mantener un orden estratégico en sus actividades diarias, permitiéndoles mantener una adecuada administración.
Según Estupiñán-Gaitán (2015) el control interno incluye el plan de organización y los procedimientos 0 metodologías que garantizan la protección de los activos, la confiabilidad de los registros contables y el cumplimiento de los lineamientos establecidos por la alta directiva de la entidad o empresa. Por tanto, este manuscrito se basa en una revisión bibliográfica del control interno aplicado en pequeñas y medianas empresas.

\section{Control interno}

Un sistema de control interno abarca aquellos aspectos que se relacionan directamente con la función de los departamentos de contabilidad y finanzas para incluir el control de presupuestos, costos estándar, reportes periódicos relacionados con las operaciones y análisis estadísticos. En este sentido, el control interno faculta a las organizaciones a llevar una planificación estandarizada que les permite alcanzar los objetivos establecidos, en función del cumplimiento de parámetros basados en normativas $y$ procedimientos que permiten satisfacer las necesidades propias de la organización (Mantilla, 2013). 
Un adecuado control interno fomenta la evaluación periódica, sistemática y constante de la organización en función de parámetros como productividad y eficiencia (Chumpitaz, 2015).

Macías et al., (2016) mencionan señalan que el control interno se ha institucionalizado y es un requisito obligatorio de las instituciones públicas ecuatorianas, no obstante, las empresas privadas se rigen en una situación diferente, debido a que no esta medida no es obligatoria para estas empresas.

La implantación del control interno se realiza mediante un sistema integrado que involucra diversos aspectos, tales como: principios, normativas, procedimientos $y$ metodologías. En base a estos principios se ven afectadas la gestión financiera que contribuye a mitigar los riesgos que inciden sobre el patrimonio, la gestión administrativa de los procesos, la confiabilidad, y oportunidad de la información, la comunicación y el cumplimiento de las leyes, políticas y regulaciones establecidas (Ricard, 2017, pp. 16).

Según Camacho-Villota et al., (2017) el control interno es un proceso continuo y sistemático efectuado por la alta directiva y los demás funcionarios o empleados de la entidad, para garantizar el cumplimiento razonable de los siguientes objetivos:

- Promover la eficiencia, eficacia y economía en las operaciones y calidad en los servicios.

- Proteger y custodiar los recursos de la entidad contra pérdidas, uso indebido, irregularidad o actos de carácter ilegal.

- Dar cumplimiento a las normativas, leyes, reglamentos y otras normas reguladoras en concordancia con las actividades desempeñadas por la organización.

- Gestionar la información financiera valida y confiable. Cabe señalar que la información debe estar debidamente documentada, como un mecanismo de respaldo frente a auditorías.

\subsection{Importancia}

Para las empresas modernas juega un rol trascendental el control interno puesto que permite definir normas y procedimientos para el correcto funcionamiento de la organización 
en el medio en el que se desenvuelve.

\subsection{Objetivos del control interno}

Camacho-Villota et al., (2017) propone los siguientes objetivos:

- Garantizar la fiabilidad y veracidad de los datos contables y extracontables.

- Gestionar el cuidado de los recursos de la empresa disminuyendo los riesgos por pérdida de información y fraudes.

- Fomentar la eficiencia en el uso de los recursos con los que dispone la empresa, bajo conceptos de sostenibilidad y sustentabilidad desde las aristas productivas, técnicas, empresariales y de manejo administrativo.

- Promover el seguimiento de las tareas o actividades propuestas desde la administración.

- Promover y evaluar constantemente la gestión administrativa, seguridad, calidad de procesos y mejora continua.

- Establecer estrategias para prevenir o disminuir los riesgos, a partir de controles que permitan detectar las desviaciones 0 perturbaciones en el manejo de las finanzas de la organización.

- Garantizar que la organización o empresa disponga de procesos de planeamiento y procedimientos adecuados para el diseño y crecimiento en la organización de procesos y procedimientos.

\section{Componentes del control interno}

El control interno incluye hasta cinco componentes, que se generan de los procedimientos mediante los cuales la administración maneja la organización. Se clasifican en: i) ambiente de control, ii) evaluación de riesgos, iii) actividades de control, iv) información y comunicación, v) supervisión y seguimiento.

El control interno no se caracteriza por ser un proceso secuencial, en donde alguno de los factores incide solamente al siguiente, sino más bien se trata de un proceso multidireccional repetitivo y permanente, en el que más de un factor afecta en los demás y en conjunto forman un sistema integrado que es dinámicamente a estable (Estupiñán-Gaitán, 2015). 
La aplicación de estos componentes es de carácter fundamental y su adecuado cumplimiento provoca mejores desempeños a nivel organizacional y el manejo administrativo de la organización.

El componente de identificación y evaluación de riesgos permite analizar los riesgos y su probabilidad de ocurrencia en el contexto de las organizaciones y en lo que cabe en sus actividades, por tanto, es de carácter imperioso contar con herramientas disponibles para este fin, independientemente de su naturaleza interna o externa. Los riesgos deben ser identificados $y$ evaluados a partir de un procedimiento estandarizado que debe ser documentado.

Las actividades de control son todas aquellas políticas y procesos diseñados para garantizar el cumplimiento del plan administrativo propuesto para un determinado período de tiempo, así como la gestión de las acciones requeridas para orientar los procesos hacia la consecución de los objetivos propuestos (Mantilla, 2013).

Los sistemas de información están diseminados en todo el ente y todos ellos atienden a uno o más objetivos de control. Los controles generales y controles de aplicación que se aplican a los sistemas de información resultan necesarios para efectuar un correcto seguimiento de las actividades realizadas (Estupiñán-Gaitán, 2015).

Desde la arista de la supervisión y seguimiento, Vega y Nieves (2016) mencionan que el monitoreo y seguimiento son estrategias políticas que forman parte del ambiente relacionado con la toma de decisiones, por consiguiente, presenta un mecanismo de carácter administrativo o en su defecto, político. Se fundamenta en la evaluación continua que debe tener el desempeño de control interno para llevar a cabo la medición de la eficiencia en el manejo de la administración.

El componente de utilidad se relaciona con el interés que se obtiene de una actividad comercial en función de los precios que se establecen en un bien o servicio que las empresas ofertan en un determinado mercado (Vom-Mises, 2016).

Por su parte, los objetivos empresariales guardan estrecha relación con la misión y visión de la 
empresa, sin interferencia de su tamaño o características tipológicas que definen la estructura empresarial. Un objetivo es una meta que debe estar delimitada en el tiempo, por lo tanto, todos los esfuerzos a nivel empresarial deben encaminarse hacia la obtención de un resultado específico. A nivel empresarial, los objetivos son los propósitos, que tomados en conjunto definen su propia razón de ser 0 existir.

El plan de objetivos puede definirse a partir de aspectos amplios, tales como mantener costos financieros y elevar el desempeño productivo e índice de liquidez de la empresa, potenciar la franja de participación en los consumidores de los productos o servicios ofertados, mejorar la producción el mínimo consumo de recursos disponibles, disminuir los costos operativos (Chiavenato, 2017).

También es válido mencionar que las alianzas estratégicas son mecanismos de cooperación empresarial, por las que varias organizaciones acuerdan voluntariamente compartir recursos para desarrollar actividades con potenciales ventajas competitivas para todas las partes convenio.

\section{Conclusiones}

Se concluye que el control interno consiste en un proceso cuya aplicación garantiza la eficiencia de las actividades y operaciones que se desarrollan en la empresa o institución, además, se basa en la aplicación de normas y políticas, que buscan dar cumplimiento a los objetivos planteados desde la alta gerencia. Por consiguiente, es imperiosa la necesidad de fomentar una estructura de control interno que se encuentre orientada a mantener la efectividad de los procesos, en riguroso apego a las leyes y normativas aplicables en función de la razón social y actividades desarrolladas por la organización.

Finalmente, se recomienda considerar la aplicación de un manual para el proceso de control interno con el fin de mejorar los procedimientos del área de ventas y así obtener resultados confiables que permitan conocer los riesgos a los que está sometida la empresa, generando una cultura de control y seguimiento para garantizar la mejora continua. 


\section{Bibliografía}

Andrés, A. F., Batista, Y. P., \& Arreola, R. J. (2014). Una propuesta normativa de control interno para las pymes mexicanas. Revista Cubana de Contabilidad y Finanzas. COFIN HABANA, (3), 77-85.

Camacho-Villota, W. A., Gil Espinoza, D. J., \& Paredes Tobar, J. A. (2017). Sistema de control interno: Importancia de su funcionamiento en las empresas. Observatorio de la Economía Latinoamericana, Ecuador.

Chiavenato, I. (2017). Administración, proceso administrativo. México: Planeación industrial

Chumpitaz, D. O. (2015). Caracterización del control interno en la gestión de las empresas comerciales del Perú 2013. In Crescendo, 6(1), 64-73.

Estupiñán-Gaitán, R. (2015). Control interno y fraudes: análisis de informe coso I, II y III con base en los ciclos transaccionales. Bogotá: Ecoe Ediciones.

Guachi, D. (2017). Análisis del Sistema de Control Interno y su Impacto en el Proceso de Ejecución Presupuestaria del Gobierno Autónomo Descentralizado del Cantón Jipijapa. Tesis de pregrado en
Ingeniería en Auditoría, UNESUM. Jipijapa-Manabí

Macías, J. M. U., Cevallos, C. D. T., \& Intriago, M. M. Z. (2016). El control interno como herramienta eficiente en las Pymes de la ciudad de Portoviejo. ECA Sinergia, 7(2), 6-15.

Mantilla, S. (2013). Auditoría de Control Interno. Bogotá: Ecoe Ediciones.

Nagua, M., \& Burgos, J. (2016). El Control Interno en las pymes familiares, una oportunidad para mejorar la competitividad empresarial. Revista Caribeña de Ciencias Sociales, (2016_09).

Ricard, D. M. (2017). Procedimiento metodológico para diseñar el sistema de control interno con enfoque por procesos en universidades cubanas. Tesis presentada en opción al grado científico de Doctor en Ciencias Contables y Financieras. Facultad de Contabilidad y Finanzas. Universidad de la Habana. La Habana, Cuba.

Vega-de la Cruz, L. O., \& NievesJulbe, A. F. (2016). Procedimiento para la Gestión de la Supervisión y Monitoreo del Control Interno. Ciencias Holguín, 22(1), 50-68.

Vom-Mises, L. (2016). Opinión de utilidad y pérdida. Estudios Públicos, 40. 